\title{
Particle-by-particle in situ characterization of the protein corona via real-time 3D single particle tracking microscopy
}

\author{
Xiaochen Tan and Kevin Welsher*
}

X. Tan, Dr. K. Welsher, Department of Chemistry

Duke University

Durham, North Carolina, 27708, USA

E-mail: kevin.welsher@duke.edu

\begin{abstract}
Nanoparticles (NPs) adsorb proteins when exposed to biological fluids, forming a dynamic protein corona that affects their fate in biological environments. A comprehensive understanding of the protein corona is lacking due to the inability of current techniques to precisely measure the full corona in situ at the single particle level. Herein, we introduce a 3D real-time single-particle tracking spectroscopy to "lock-on" to single freely-diffusing polystyrene NPs and probe their individual protein coronas. The diffusive motions of the tracked NPs enable quantification of the "hard corona" using mean-squared displacement analysis. Critically, this method's particle-by-particle nature enabled a lock-in-type frequency filtering approach to extract the full protein corona, despite the typically confounding effect of high background signal from unbound proteins. From these results, the dynamic in situ full protein corona is observed to contain double the number of proteins than are observed in the ex situ measured "hard" protein corona.
\end{abstract}

\section{Introduction}

Nanoparticles (NPs) have become increasingly important in biomedical research, including drug delivery, diagnostics, cellular imaging, and phototherapies. ${ }^{[1-4]}$ However, when exposed to biological fluids, NPs immediately adsorb free proteins onto their surfaces, forming a dynamic protein layer called the "protein corona".[5] It has been well recognized that this protein corona alters the size, ${ }^{[6,7]}$ dispersion, ${ }^{[8-10]}$ and surface chemistry of NPs, ${ }^{[11,12]}$ leading to unpredictable therapeutic behaviors in biological systems. ${ }^{[11,13-18]}$ Surface modification with zwitterionic ligands or polyethylene glycol has been shown to reduce protein adsorption, although adsorption cannot be completely suppressed. ${ }^{[19]}$ In recent years, other methods have been developed that, instead of preventing adsorption, aim to control and modify this protein layer for its potential biomedical applications. ${ }^{[20,21]}$ Understanding the formation of this complex and dynamic protein corona is critical for understanding and ultimately predicting the biological behaviors of NP-protein complexes.

Protein corona adsorption is a multi-faceted process, affected by the diversity of both proteins and nanomaterials, concentration, surface functionalization, and a host of other factors. ${ }^{[12,22-24]}$ Efforts to characterize protein corona formation can be divided into two classes: ex situ techniques and in situ techniques. ${ }^{[25,26]}$ The "hard" protein corona, wherein proteins are adsorbed onto NPs with large adsorption binding energy $\left(\Delta \mathrm{G}_{\mathrm{ads}}\right)$, remain bound to the particle after it has been separated from its native environment. The 
removal of the NP from the protein solution allows further protein composition analysis using ex situ methodologies such as SDS/PAGE and LC/MS. However, the extent to which separation methods that remove nanoparticles from the biological fluid alter the protein adsorption equilibrium remains unknown. Moreover, it is suggested that some of the tightly bound interfacial proteins cannot be detached by general methods ${ }^{[27]}$, and their influence on NP agglomeration is also not well understood. In situ techniques such as fluorescence correlation spectroscopy $(\mathrm{FCS})^{[6,7]}$ and dynamic light scattering $(\mathrm{DLS})^{[10,23]}$ characterize not only the "hard" protein corona, but also loosely bound and highly dynamic "soft" corona in native protein solution by measuring the hydrodynamic radius change due to protein binding. Unfortunately, these ensemble techniques provide limited information for quantitative protein corona measurement because they fail to reveal the direct interactions between the proteins and NPs, and may conflate protein corona size and NP aggregation. At their core, both FCS and DLS rely on changes in diffusion coefficient to determine the size of a NP-protein complex. This is particularly challenging for therapeutically relevant nanoparticles at sizes of $100 \mathrm{~nm}$ or greater, where even large changes in the quantity of proteins in the protein corona lead to small changes in the hydrodynamic radius. To overcome the intrinsic uncertainty in diffusion-based in situ methods, recent studies have utilized imaging-based strategies to directly observe single NP-protein interactions with high sensitivity. ${ }^{[8,28-31]}$ While far more sensitive than extracting a diffusion coefficient, each of these methods required immobilization of the NP for sufficient characterization. The change of dynamics of the NP and the interaction between NP and surface may strongly affect protein binding, and it is difficult, if not impossible, to distinguish NP-bound proteins from surface-bound proteins. Accordingly, a highly sensitive and non-perturbative method that can access individual particle-protein interaction in situ without surface tethering is in enormous demand, owing to the complexity of highly dynamic NP-protein interactions and the diversity of nanomaterialbiological system. Probing these NP-protein interactions in situ can finally dissect the relative abundance of the hard and soft protein corona.

Real-time 3D single-particle tracking (RT-3D-SPT) spectroscopy removes the surface-tethering requirement of imaging-based methods while preserving single-particle sensitivity, due to its ability to capture and analyze individual freely diffusing nanoscale object in situ. ${ }^{[32-34]}$ Herein, we introduce an RT-3D-SPT-based lock-on protein corona spectroscopy to quantify the proteins adsorbed onto freely diffusing polystyrene nanoparticles (PS NPs), employed here as a model system. This microscopy, called 3D Single-Molecule Active Real-time Tracking (3D-SMART), ${ }^{[35,36]}$ uses optical feedback to lock-on to a single freely diffusing PS NP-protein complex in three dimensions to continuously and simultaneously monitor the fluorescence signals from both the PS NP and their associated protein coronas (Figure 1, Figure S1-5, Movie S1). This continuous observation enables real-time characterization and unlocks a wealth of information. The diffusive motions of the particle yield information on protein adsorption-induced changes in the hydrodynamic radius, while the fluorescence intensity from NP-bound proteins enables quantitative hard corona analysis on a per-particle basis with single protein sensitivity. Critically, an entirely in situ analysis of the full corona (hard plus soft) via a lock-in type filtering algorithm allowed us to directly measure the full protein corona on a single nanoparticle in solution, despite the confounding effects of large signals from unbound "background" proteins. This methodology, which can be extended to other 
nanomaterial-protein systems, allows precise and sensitive determination of individual protein corona without removing the NP or protein from its native solution, opening the possibility to study transient and dynamic protein-nanoparticle interactions which are destroyed by current ensemble and surface-tethered imaging techniques.

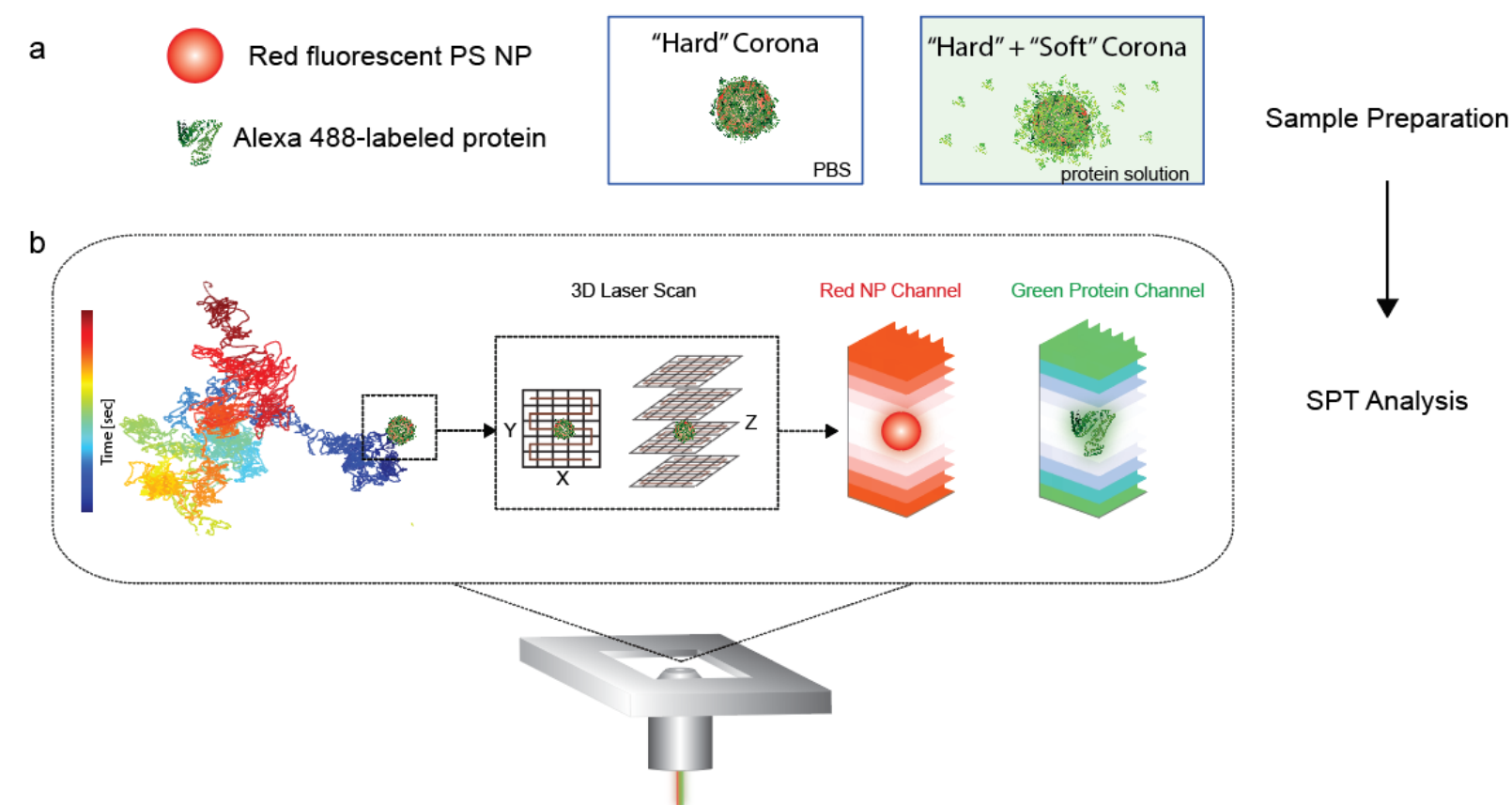

Figure 1. Schematic illustration of 3D-SMART used for in-situ observation of protein corona. (a) Red fluorescent PS NPs are incubated with Alexa 488-labeled protein solution. The hard corona and full (hard+soft) corona are quantitatively analyzed by the 3D-SMART microscope. (b) Individual NP-protein complexes are "locked on" to the center of 3D laser scanning volume. The NP trajectories (left) and emissions of both red NP and green protein channels (right) are recorded simultaneously for consequent protein corona analysis.

\section{Results and Discussion}

\section{Protein Corona Measurement via NP Sizing}

Each particle captured and analyzed by the 3D-SMART microscope yields XYZ position information, sampled every $10 \mu \mathrm{sec}$ for on the order of a minute-long at a time (Mean $\pm S D=51.3 \pm 21.5 \mathrm{~s}$, Figure S3). We first investigated the effect of protein corona formation on NP size particle by particle using mean square displacement (MSD) analysis on these XYZ NP-corona complex trajectories. Red fluorescent polystyrene (PS) NPs (Bangs Labs) with a diameter of approximately $190 \mathrm{~nm}$ were chosen as model system, and transmission electron microscopy (TEM) images revealed uniform size distribution of these bare PS NPs (Figure S6). Individual PS NP-protein complexes were tracked and analyzed by 3D-SMART to monitor the formation of the protein corona, using the obtained $3 \mathrm{D}$ trajectories to calculate changes in hydrodynamic radius (Figure 2a). To compare different protein environments, PS NPs were incubated for $30 \mathrm{~min}$ in solutions containing different concentrations of BSA $(0.0083 \mathrm{mg} / \mathrm{mL}, 0.33 \mathrm{mg} / \mathrm{mL}, 0.90 \mathrm{mg} / \mathrm{mL}, 1.4 \mathrm{mg} / \mathrm{mL}$, $1.7 \mathrm{mg} / \mathrm{mL})$. The hydrodynamic radius $\left(\mathrm{R}_{\mathrm{H}}\right)$ of each NP complex was then extracted by performing MSD analysis and applying the Stokes-Einstein equation to the resulting 
diffusion coefficient (Figure 2b). Tracked particles exhibited a linear relationship between MSD and time, indicating Brownian diffusion $\left(\mathrm{R}^{2}>0.95\right.$, Figure S7). The hydrodynamic radii of PS NP-protein complexes incubated in various BSA solutions were quantified for a large number of trajectories ( $n>50$, Figure S8). Figure $2 c$ demonstrates representative size histograms of bare PS NPs $(n=75)$ and PS NPs $(n=73)$ trajectories after incubating with $1.7 \mathrm{mg} / \mathrm{mL}$ of BSA solution, showing that the average size changed from $82.4 \pm 21.3$ $\mathrm{nm}$ (expressed as mean $\pm S D$ ) to $93.8 \pm 32.6 \mathrm{~nm}(\mathrm{p}=0.01$, two-tailed t-test). From the size distribution, the PS NPs exposed to protein lead to a few very large NP complexes, which could be attributed either to NP aggregation or formation of the protein corona. Similar results were also observed by dynamic light scattering (DLS) control experiments (Figure 2d, Figure S8, Table S1). To determine if the apparent difference in hydrodynamic radius was simply due to NP aggregation, we differentiated, characterized, and excluded the protein-mediated NP aggregate trajectories by setting a threshold for PS NP intensity to distinguish between single particles and aggregates (Figure S9). Following removal of these "bright" NP aggregates from the data set, only a modest increase of average hydrodynamic radius $\left(R_{H}\right)$ was observed from bare PS NPs $(81.3 \pm 20.1 \mathrm{~nm}, \mathrm{n}=73)$ to PS NPs exposed to $1.7 \mathrm{mg} / \mathrm{mL}$ BSA $(83.5 \pm 20.5 \mathrm{~nm}, \mathrm{n}=60)$. In fact, the null hypothesis that there is no change in hydrodynamic radius upon exposure to protein solution could not be rejected for any protein concentration tested $(p>0.05$, distributions of other protein concentrations shown in Figure S10). This suggests that the NP complex varies little in size before and after protein incubation and that this size change is below the detection limit that MSD analysis can provide, owing to the intrinsic noise associated with measuring diffusion coefficients. We also performed MSD sizing analysis on carboxyl functionalized PS-COOH NP. However, NP clusters with noticeably large $\mathrm{R}_{\mathrm{H}}$ were found when exposed to protein solutions, which hindered further single PS-COOH NP-protein complex analysis using sizing data alone. (Figure S11).

We next investigated sizing change on PS NP-hard corona complexes by extracting PS NPs from the protein solution. Unlike the in situ measurement described above, the separation process (three centrifugation/washing steps) removes any proteins that are not tightly bound to the PS NPs. In good agreement with the in-situ measurements above, no significant change in size of single PS NP-hard corona was observed as a function of protein concentration or incubation time after excluding NP aggregate trajectories (Figure S12).

The failure of both MSD and DLS measurements to sensitively quantify changes in NP-corona is not surprising, given that both rely on a measurement of particle diffusion for sizing. Even though 3D-SMART can distinguish single particles from aggregates, which can be a confounding factor in DLS, the considerable uncertainty associated with a diffusion-based result makes quantification impossible. Additionally, the degree of aggregation increased by more than three-fold when excess proteins were removed, inversely related to incubation time or the presence of other serum proteins (Figure S13). This NP aggregation could be explained by previous work, suggesting that sparsely adsorbed proteins undergo structural changes that lead to NPs dimers. ${ }^{[8]}$ Fewer aggregates were found when the experiment was repeated with immunoglobulins (IgG) instead of BSA (Figure S11,13), indicating that protein-mediated aggregation is also determined by protein type at the same protein concentration. 

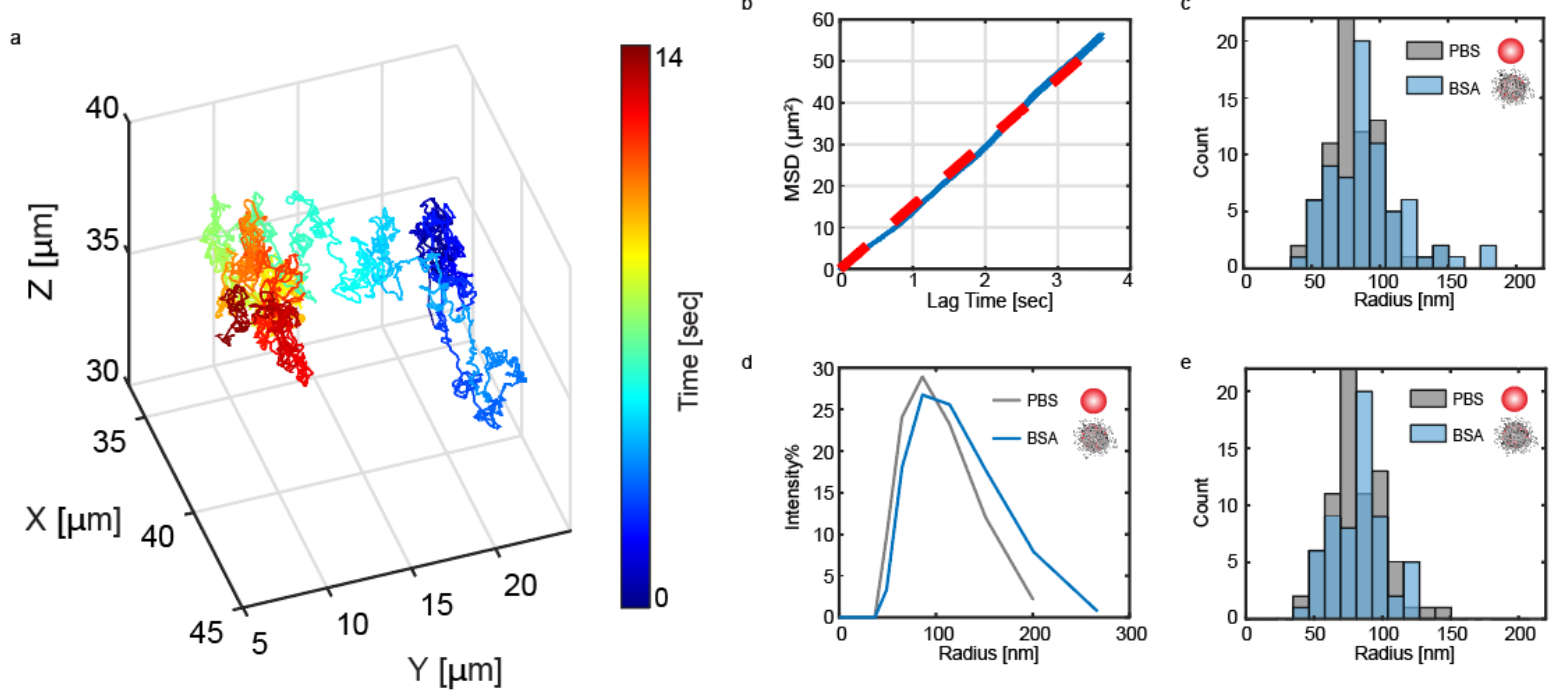

Figure 2. Sizing measurement of NP-protein corona. (a) Example 3D trajectory of single PS NP-protein complex obtained by 3D-SMART. (b) MSD analysis of (a). The slope of the linear fit (red) from the MSD plot (blue) yields a diffusion coefficient of $\mathrm{D}=2.5 \mu \mathrm{m}^{2} / \mathrm{s}$. (c) Radius histograms of PS NP complex before $\left(\mathrm{R}_{H}=82.4 \pm 21.3 \mathrm{~nm}, \mathrm{~N}=75\right.$, grey) and after $\left(R_{H}=93.8 \pm 32.6 \mathrm{~nm}, \mathrm{~N}=73\right.$, blue) 30 min incubation with $1.7 \mathrm{mg} / \mathrm{mL}$ of BSA solution. (d) DLS measurement of PS NP complexes (Grey: PS NPs in PBS buffer; blue: PS NPs after 30 min incubation with $1.7 \mathrm{mg} / \mathrm{mL}$ of BSA). (e) Radius histograms of (c) after removal of NP aggregation. The $R_{H}$ is expressed as mean $\pm S D$.

\section{Hard Corona Quantification}

We have demonstrated that sizing data alone fails to even qualitatively resolve the protein corona formation of $190 \mathrm{~nm}$ PS NPs, let alone quantify both the hard and soft corona's contributions. This is where the ability to lock-on to a single nanoparticle becomes extremely valuable. In addition to translational behavior used above, 3DSMART also collects intensity information from both the NP and the protein layer (if appropriately labeled), enabling corona proteins to be precisely quantified on freely diffusing NPs.

First, we investigated the hard corona proteins by tracking PS NP-protein complexes separated from Alexa 488-labeld BSA solutions by a series of centrifugation and washing steps (See Supplementary Information). The washing steps removed the proteins in the loosely bound soft corona, meaning the protein signal collected arose only from hard corona proteins (Figure S14). To quantify the number of hard corona proteins in an individual complex, we used the initial protein intensity (indicated by the red arrow in Figure 3a, left), Is, by equation 1 :

$$
\mathrm{N}_{\text {Protein }}=\frac{\mathrm{I}_{\mathrm{S}}}{\mathrm{DOL} * \mathrm{I}_{0}}
$$

with degree of labeling, DOL, and single dye signal, Io. The DOL was obtained using the dye-to-protein ratio obtained from bulk UV-Vis measurements (Figure S15). The single Alexa 488 intensity, Io, was estimated by immobilizing Alexa 488-labeled NPs onto a coverslip and measuring the intensity drop of the last photobleaching step (Figure S16). 
Following equation 1, PS NPs incubated in $0.32 \mathrm{mg} / \mathrm{mL}$ of Alexa 488-BSA solution for 1 min were observed to have $142 \pm 5$ (mean \pm SE) BSA per PS NP (Figure 3a). This number rose to $243 \pm 7$ proteins at $16 \mathrm{~h}$ incubation time (Figure $3 \mathrm{~b}$, blue bars). The time evolution at all three tested BSA concentrations $(0.32 \mathrm{mg} / \mathrm{mL}, 0.90 \mathrm{mg} / \mathrm{mL}, 1.4 \mathrm{mg} / \mathrm{mL})$ indicates that hard corona formation is a rapid process in which more than half of the tightly bound proteins are adsorbed within just $1 \mathrm{~min}$ (Figure $3 \mathrm{~b}$ ). The number of tightly bound proteins at equilibrium (16 h) was estimated to be $243 \pm 7,488 \pm 8$ and $625 \pm 11$ for $0.32 \mathrm{mg} / \mathrm{mL}$, $0.90 \mathrm{mg} / \mathrm{mL}$, and $1.4 \mathrm{mg} / \mathrm{mL}$ of BSA concentrations, respectively. These values are much smaller than the expected saturated monolayer protein quantity $N_{s a t}=3276$ (for BSA triangular face binding, $\mathrm{N}_{\text {sat }}=3243$ for BSA rectangular face binding, see Supplementary Information). The fact that the measured number of proteins in the hard corona is far smaller than this value indicates a sub-monolayer of hard corona proteins. The resulting bare NP surfaces may contribute to protein-mediated NP-NP interaction during centrifugation, evidenced by the increase in NP aggregates observed after purification. To further investigate the effect of separating the NPs from the protein solution, we plotted the number of hard corona proteins against NP fluorescence (Figure 3d, red dotted line is the threshold of NP aggregation). The linear correlation suggests a consistent hard corona protein quantity per NP, indicating that centrifugation does not strongly affect the hard corona composition.

Finally, to ensure that proteins were not desorbing from the NP surface during purification, we compared the BSA hard corona quantity $(0.32 \mathrm{mg} / \mathrm{mL}, 16 \mathrm{~h}$ incubation time) after both two and three washing steps by PBS. No significant difference $(p>0.05$, two-tailed t-test) in protein adsorption was observed, indicating no loss on the hard corona due to successive washing steps (Figure S17). To further test the stability of the hard corona after purification, we plotted the observed hard protein quantity versus trajectory number over the more than an hour-long tracking experiment (Figure 3c). Other than particle-to-particle variance in the number of proteins per corona, no overall trend was observed in the average number of proteins as a function of time (Figure S18). These results suggest that these hard corona proteins are highly stable and likely remain bound to the nanoparticle in vivo, despite the shifted equilibrium following removal of the protein solution.

It has been well established that the affinity of proteins for a nanoparticle surface varies widely. To probe whether the protein type affects hard protein corona formation, we also performed the same hard corona experiment on IgG. Interestingly, the hard corona protein quantity decrease from $243 \pm 7$ to $74 \pm 3$ for $\mathrm{lgG}$ hard corona under same experimental condition ( $5.4 \mu \mathrm{M}, 16 \mathrm{~h}$ incubation time) even though IgG has been reported to have a higher binding affinity than BSA (Figure 3e, Figure S19). [37]

While single component BSA or IgG solutions serve as a useful model system, a real analysis of the protein corona must be done in a more complex environment. Fetal bovine serum (FBS) comprises a variety of distinct proteins that compete to bind to nanoparticles, depending on their abundance and binding affinities. According to the Vroman effect, it has been proposed that the most abundant proteins such as BSA are adsorbed first and displaced by proteins with higher binding affinity. ${ }^{[38]}$ However, in recent years there have been contradictory reports regarding irreversibly bound proteins. ${ }^{[39]}$ To test how much of the protein corona we observe here is irreversibly bound, we compared the BSA hard corona formation in single-component solutions and in FBS with the same BSA 
concentration (20.6 $\mu \mathrm{M}$, see Supplementary Note 1). In partial agreement with the Vroman effect, only $\sim 15 \%$ replacement of "hard" BSA was observed in the presence of other bovine serum proteins, which is contrary to previous studies suggesting a significant decrease (Figure S20). [29] This suggests that the hard corona is largely irreversibly bound.

We note here that the above-described analysis applies to nanoparticles that have been incubated in labeled protein solution and then purified via centrifugation, similar to other ex situ techniques. A complete quantification of the full corona requires an in situ measurement, readily available via 3D-SMART and described below.

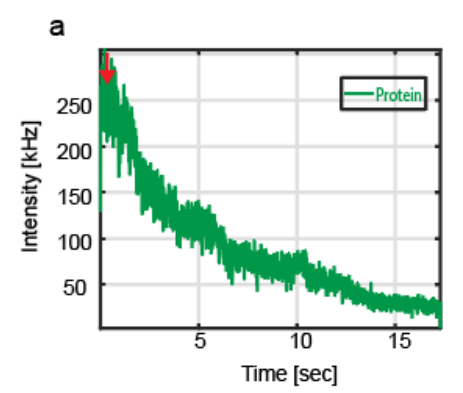

b

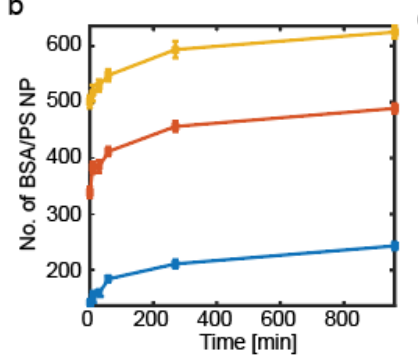

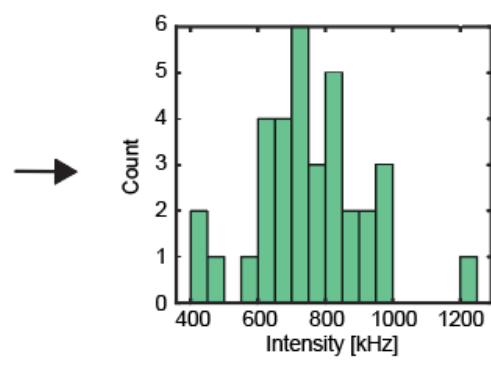

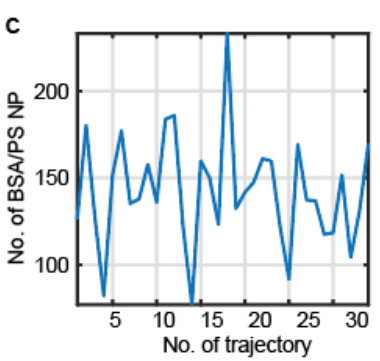

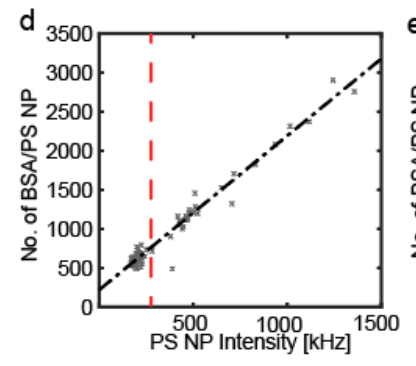

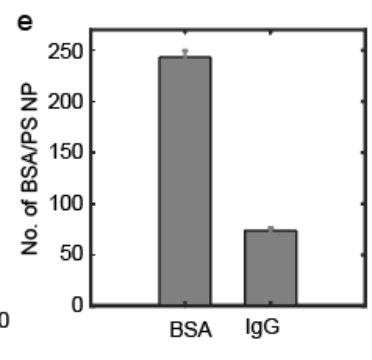

Figure 3. (a) Example intensity trace of hard corona in $0.32 \mathrm{mg} / \mathrm{mL}$ BSA solution (left), intensity distribution (middle) and protein quantity distribution (right) of 34 trajectories. (b) protein corona time evolution in $0.32 \mathrm{mg} / \mathrm{mL}$ (blue), 0.90 $\mathrm{mg} / \mathrm{mL}$ (red), $1.4 \mathrm{mg} / \mathrm{mL}$ (yellow) of BSA solutions. (c) Example time trace of protein quantity within $1 \mathrm{~h}$ experimental tracking time $(0.32 \mathrm{mg} / \mathrm{mL}$ BSA solution, $16 \mathrm{~h}$ incubation). (d) The number of BSA per PS NP complex plotted against PS NP intensity. The red dashed line indicates intensity threshold for single NP and aggregations (e) BSA hard corona vs. IgG hard corona $(5.4 \mu \mathrm{M})$.

\section{In situ measurement of the full (hard plus soft) corona}

Due to the difficulties in preserving the highly dynamic "soft" corona upon extracting NPs from the biological fluid, very few studies have been done to directly monitor these interactions. ${ }^{[29,31,40]}$ To observe this soft corona using fluorescence, all proteins in the solution need to be fluorescently labeled, creating an enormous background signal, particularly at biologically relevant protein concentrations, burying the far smaller corona signal in the noise (Figure $4 \mathrm{a}, \mathrm{c}$ ). Some previous imaging studies employed a partial labeling strategy $(\sim 1 \%)$ that may generate significant soft corona quantification error and is limited to low and physiologically irrelevant protein concentrations. ${ }^{[10,29]}$ The ability to lock-on to the diffusing nanoparticle presents a unique opportunity to distinguish the small soft corona signal from the overwhelming background signal. To do so, we applied a lockin type filtering algorithm to extract the indistinguishable corona signal in the high background environment of fluorescently labelled proteins. As shown in Figure 4a, the protein signal is primarily located in the center of the laser scanning pattern, whereas the background signal is evenly distributed over the entire pattern. As a result, the time- 
dependent variation in the signal from the protein corona should differ from the signal from background proteins. This is evidenced by characteristic peaks corresponding to the NP-protein complex in frequency space. Among the several peaks observed in the frequency spectrum, the peak at $10 \mathrm{kHz}$ was found to have the highest sensitivity to intensity in both simulated and experimental data (Figure 4b, Figure S21, 22). The amplitude of the $10 \mathrm{kHz}$ signal increases linearly with the particle intensity, meaning that it can be used to quantitatively characterize the in situ protein corona (i.e., hard plus soft corona), even in the extremely high background environment of freely diffusing and fluorescently labeled BSA. We note here that the solution phase protein displayed a small residual background at $10 \mathrm{kHz}$. This background was calibrated as a function of protein concentration and subtracted from the signal for each experiment (Figure 4b, d).

Figure 4c shows example intensity traces of red PS NP and green protein channels where tracking was initiated approximately in the middle of the time course. There is no visible change in the average intensity of the protein signal upon initiation of tracking, aside from small fluctuations caused by the particle complex moving deeper into the sample. Figure 4d shows the corresponding lock-in amplitude of BSA proteins at $10 \mathrm{kHz}$, in which the amplitude increased from $17167 \pm 298$ a.u. (tracking off, background only) to $21509 \pm 802$ a.u. (tracking on, background plus protein corona signal), indicating formation of the protein corona. To validate that this approach could extract small signals bound to diffusing nanoparticles in the presence of large background signals, we studied the interaction between PS NPs and fluorophores by incubating PS NPs in $15 \mu \mathrm{M}$ of Alexa 488 and Atto 655 solutions. In comparison to just background amplitude without tracking, we observed an increase of the lock-in amplitude of Atto 655 when tracking PS NPs (1970 \pm 1302 a.u., Figure 4e, red bar), which is impossible to measure simply from the via fluorescent intensity in the presence of $2 \mathrm{MHz}$ background intensity. The increase of lockin amplitude when tracking PS NP in Atto solution demonstrates adsorption of Atto 655 occurred in the absence of proteins, suggesting that this type of dye is not suitable for fluorescent labeling in this study. In contrast, Alexa 488, the fluorophore chosen for this work, shows negligible adsorption onto PS NP, evidenced by the fact that the lock-in amplitude of Alexa 488 from tracked PS NPs was indistinguishable from the background $(-730 \pm 617$ a.u., green bar, Figure $4 \mathrm{e})$.

The linear correlation between the fluorescence signal and the lock-in signal was also observed in hard protein corona experiments so that the difference in amplitude can be converted into the real fluorescence signal from the particle in the presence of high background (Figure 4f). For more precise conversion, we tracked $200 \mathrm{~nm}$ multicolored beads using the red fluorescence channel and calculated their green fluorescence in a high background Alexa 488 solution to generate a calibration (Figure S23, Supplementary Note 2). This calibration experiment enabled full corona quantification and was performed before each full corona experiment. For the first time, this method was able to extract and quantify the in situ soft corona of a single, freely-diffusing nanoparticle at extremely small signal to background ratio (SBR). Our results showed that the full corona consisted of $734 \pm 19$ BSA per PS NP. Using this value, we can then deduce the quantity of soft corona proteins by comparing to the value measured for the same particles under the hard corona condition (particles extracted from the protein solution). Under the same conditions (1.0 $\mathrm{mg} / \mathrm{mL}$ of BSA solution, $16 \mathrm{~h}$ incubation) the hard corona was measured to have $366 \pm$ 11 BSA per PS NP (Figure 4g). This indicates the full corona, measured in situ, contains 
nearly double the proteins typically extracted from ex situ hard corona measurements. The lock-in filtering-based algorithm enables extracting this full corona protein signal in native protein solution at a SBR of $1.1 \%$. Simulations under same background condition that the SBR needs to be larger than $0.8 \%$ to resolve the amplitude change (i.e., $15 \mathrm{kHz}$ signal in $1.8 \mathrm{MHz}$ background). This limitation can be pushed further by altering the algorithm's cut-off frequency (Figure S24), providing possibilities to resolve even more subtle changes in protein adsorption in high background environments.

a
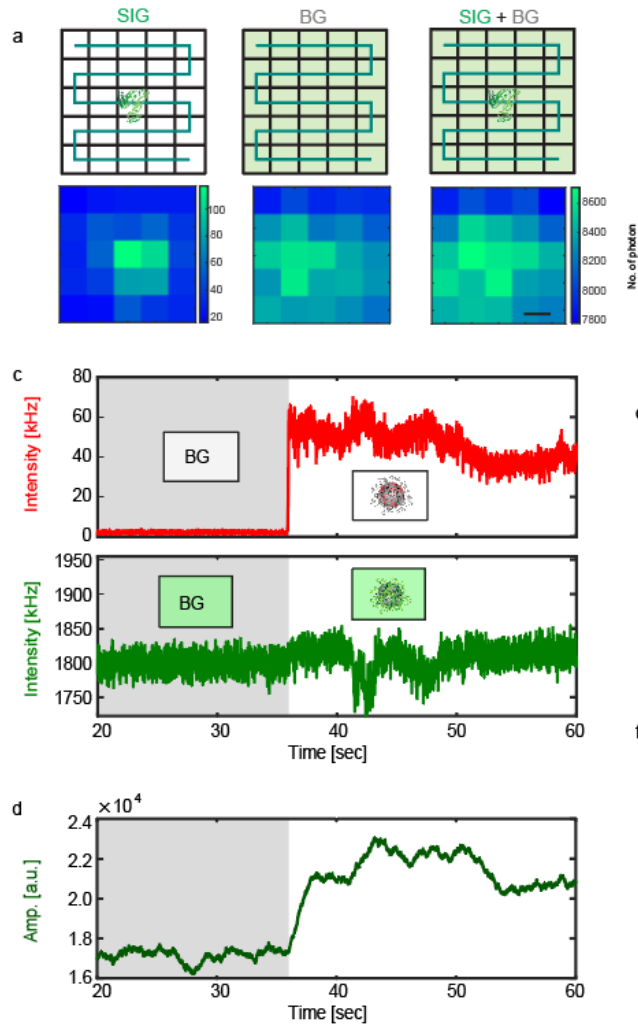
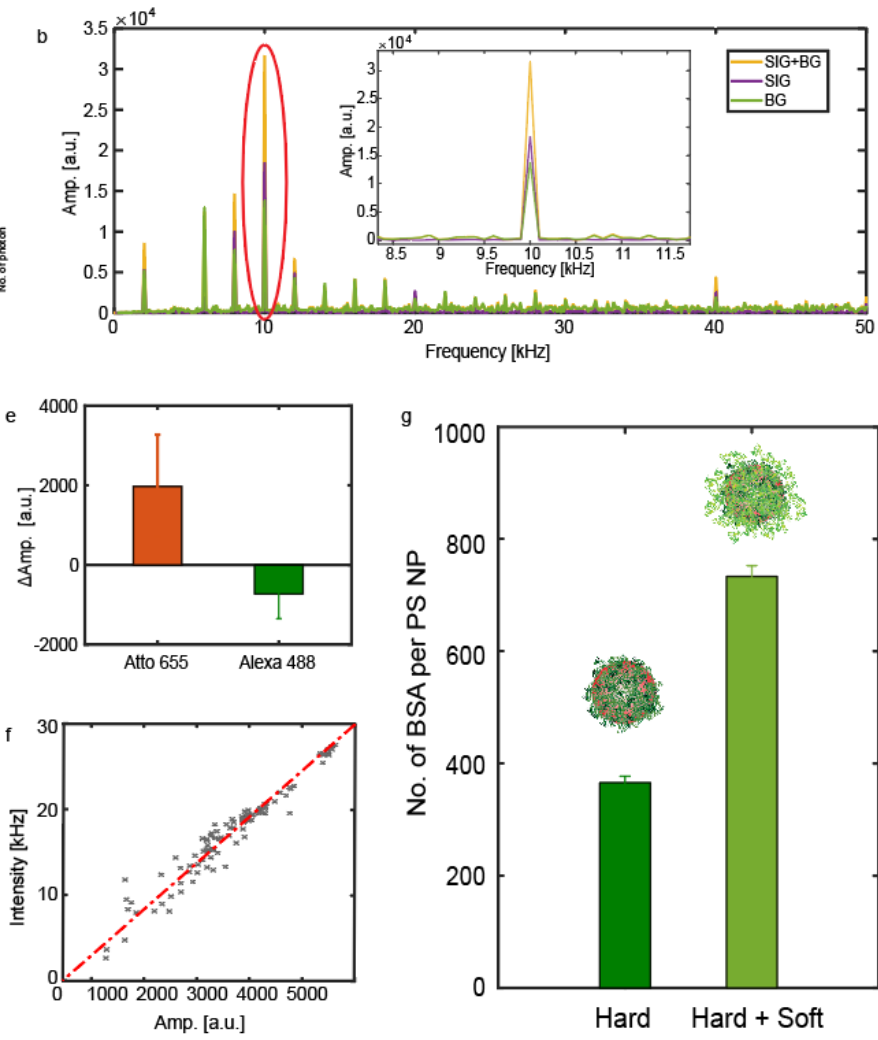

Figure 3. Lock-in type filtering algorithm extracts soft corona signal that is buried by the noise of high background. (a) Top: schematic illustration centroid proteins(left), uniform background (middle), and proteins in BG (left) in the raster laser scanning pattern. Bottom: Corresponding experimental images. Scale bar: $200 \mathrm{~nm}$. (b) The lock-in scan of PSNP (purple), Alexa 488 dye solution (green), and PSNP plus Alexa 488 (yellow) signal. The subfigure inside is the zoomin of the circled area. (c) Intensity trace of red PS NP (top) and green protein channel (bottom) from tracking off (grey background) to tracking on (white background). In-situ protein corona when tracking is on cannot be resolved just by intensity trace. (d) The $10 \mathrm{kHz}$ lock-in amplitude trace corresponding with (c). (e) $10 \mathrm{kHz}$ lock-in amplitude difference between tracking-on and tracking-off state in ATTO 655 solution (red) and Alexa 488 solution (green). (f) Experimental intensity plotted against lock-in amplitude at $10 \mathrm{kHz}$ (grey) and linear fitting (red). (g) In-situ full corona vs. hard corona protein quantity $(1.0 \mathrm{mg} / \mathrm{mL}$ of BSA solution).

\section{Conclusion}

The above work demonstrates the power of using real-time single-particle tracking to measure all aspects of the NP protein corona. In this work, we applied 3D-SMART to sensitively quantify proteins adsorbed onto freely diffusing polystyrene nanoparticles on a per-particle basis, without the disruptive use of surface tethering used in other imagingbased techniques. The simple MSD analysis indicated that for NPs at the size scale investigated here is insufficient for detecting changes in the size of the protein corona, 
outside of merely responding to changes in the aggregation state of the NPs themselves. This suggests that methods that rely on changes in diffusion coefficient alone (such as DLS and FCS) are insufficient for characterizing the protein corona in this size regime. Critically, the experiments above demonstrate that 3D-SMART massively improves the sensitivity of single-particle analysis by counting the number of individual proteins on the NP surface, something that could only previously be achieved by surface-immobilization. By locking onto individual freely diffusing NP-PC complexes, we were able to obtain the diffusion and fluorescence signal of both NP and the associated protein corona, study the time evolution of hard protein corona formation, and examine multiple influencing factors (protein concentration, protein type, presence of other proteins, etc.). Moreover, we could differentiate between the hard and soft corona quantitatively by applying a lock-in filteringbased algorithm despite the high background of freely diffusing proteins at an SBR near $1 \%$. A sub-monolayer of both hard and full (hard plus soft) corona formation was suggested from our experiment, where a roughly equal number of proteins was found in soft and hard corona, which cannot be resolved by sizing change alone. To our knowledge, this is the first quantitative observation of in situ protein corona without surface tethering or partial labeling strategies. This approach's flexibility opens the possibility to study transient protein-NP interactions that are obscured or perturbed by current ensemble and surface-tethered imaging-based methods, respectively. Relevant future work includes examining other nanomaterial-protein systems and translating this technique to the in vivo setting to further understand the effect of protein corona on nanoparticle fate.

\section{Acknowledgements}

The authors acknowledge financial support from the National Institute of General Medical Sciences of the National Institutes of Health under award number R35GM124868, and from Duke University.

\section{Reference}

[1] T. M. Allen, P. R. Cullis, Science (80-. ). 2004, 303, 1818-1822.

[2] W. H. De Jong, P. J. A. Borm, Int. J. Nanomedicine 2008, 3, 133-149.

[3] D. E. Lee, H. Koo, I. C. Sun, J. H. Ryu, K. Kim, I. C. Kwon, Chem. Soc. Rev. 2012, 41, 2656-2672.

[4] B. Pelaz, C. Alexiou, R. A. Alvarez-Puebla, F. Alves, A. M. Andrews, S. Ashraf, L. P. Balogh, L. Ballerini, A. Bestetti, C. Brendel, S. Bosi, M. Carril, W. C. W. Chan, C. Chen, X. Chen, X. Chen, Z. Cheng, D. Cui, J. Du, C. Dullin, A. Escudero, N. Feliu, M. Gao, M. George, Y. Gogotsi, A. Grünweller, Z. Gu, N. J. Halas, N. Hampp, R. K. Hartmann, M. C. Hersam, P. Hunziker, J. Jian, X. Jiang, P. Jungebluth, P. Kadhiresan, K. Kataoka, A. Khademhosseini, J. Kopeček, N. A. Kotov, H. F. Krug, D. S. Lee, C. M. Lehr, K. W. Leong, X. J. Liang, M. L. Lim, L. M. Liz-Marzán, X. Ma, P. Macchiarini, H. Meng, H. Möhwald, P. Mulvaney, A. E. Nel, S. Nie, P. Nordlander, T. Okano, J. Oliveira, T. H. Park, R. M. Penner, M. Prato, V. Puntes, V. M. Rotello, A. Samarakoon, R. E. Schaak, Y. Shen, S. Sjöqvist, A. G. Skirtach, M. G. Soliman, M. M. Stevens, H. W. Sung, B. Z. Tang, R. Tietze, B. N. Udugama, J. Scott 
VanEpps, T. Weil, P. S. Weiss, I. Willner, Y. Wu, L. Yang, Z. Yue, Q. Zhang, Q. Zhang, X. E. Zhang, Y. Zhao, X. Zhou, W. J. Parak, ACS Nano 2017, 11, 23132381.

[5] T. Cedervall, I. Lynch, S. Lindman, T. Berggard, E. Thulin, H. Nilsson, K. A. Dawson, S. Linse, Proc. Natl. Acad. Sci. 2007, 104, 2050-2055.

[6] C. Röcker, M. Pötzl, F. Zhang, W. J. Parak, G. U. Nienhaus, Nat. Nanotechnol. 2009, 4, 577-580.

[7] L. Shang, G. U. Nienhaus, Acc. Chem. Res. 2017, 50, 387-395.

[8] S. Dominguez-Medina, L. Kisley, L. J. Tauzin, A. Hoggard, B. Shuang, A. S. D. S. Indrasekara, S. Chen, L. Y. Wang, P. J. Derry, A. Liopo, E. R. Zubarev, C. F. Landes, S. Link, ACS Nano 2016, 10, 2103-2112.

[9] L. K. Müller, J. Simon, C. Rosenauer, V. Mailänder, S. Morsbach, K. Landfester, Biomacromolecules 2018, 19, 374-385.

[10] H. Wang, R. Ma, K. Nienhaus, G. U. Nienhaus, 2019, 1900974, 1-12.

[11] S. Tenzer, D. Docter, J. Kuharev, A. Musyanovych, V. Fetz, R. Hecht, F. Schlenk, D. Fischer, K. Kiouptsi, C. Reinhardt, K. Landfester, H. Schild, M. Maskos, S. K. Knauer, R. H. Stauber, Nat. Nanotechnol. 2013, 8, 772-781.

[12] G. Baier, C. Costa, A. Zeller, D. Baumann, C. Sayer, P. H. H. Araujo, V. Mailänder, A. Musyanovych, K. Landfester, Macromol. Biosci. 2011, 11, 628-638.

[13] V. P. Vu, G. B. Gifford, F. Chen, H. Benasutti, G. Wang, E. V Groman, R. Scheinman, L. Saba, S. M. Moghimi, D. Simberg, Nat. Nanotechnol. 2019, DOI 10.1038/s41565-018-0344-3.

[14] E. L. L. Yeo, P. S. P. Thong, K. C. Soo, J. C. Y. Kah, Nanoscale 2018, 10, 24612472.

[15] C. C. Fleischer, C. K. Payne, Acc. Chem. Res. 2014, 47, 2651-2659.

[16] C. C. Fleischer, C. K. Payne, J. Phys. Chem. B 2014, 118, 14017-14026.

[17] S. Lara, F. Alnasser, E. Polo, D. Garry, M. C. Lo Giudice, D. R. Hristov, L. Rocks, A. Salvati, Y. Yan, K. A. Dawson, ACS Nano 2017, 11, 1884-1893.

[18] Z. J. Deng, M. Liang, M. Monteiro, I. Toth, R. F. Minchin, Nat. Nanotechnol. 2011, 6, 39-44.

[19] J. Mosquera, I. Garciá, M. Henriksen-Lacey, M. Martínez-Calvo, M. Dhanjani, J. L. Mascarenãs, L. M. Liz-Marzán, ACS Appl. Mater. Interfaces 2020, DOI 10.1021/acsnano.9b08752.

[20] J. Y. Oh, H. S. Kim, L. Palanikumar, E. M. Go, B. Jana, S. A. Park, H. Y. Kim, K. Kim, J. K. Seo, S. K. Kwak, C. Kim, S. Kang, J. H. Ryu, Nat. Commun. 2018, 9, 19.

[21] M. Hadjidemetriou, K. Kostarelos, Nat. Nanotechnol. 2017, 12, 288-290.

[22] M. Lundqvist, J. Stigler, G. Elia, I. Lynch, T. Cedervall, K. A. Dawson, Proc. Natl. Acad. Sci. U. S. A. 2008, 105, 14265-70.

[23] G. Maiorano, S. Sabella, B. Sorce, V. Brunetti, M. A. Malvindi, R. Cingolani, P. P. Pompa, ACS Nano 2010, 4, 7481-7491.

[24] L. Treuel, S. Brandholt, P. Maffre, S. Wiegele, L. Shang, G. U. Nienhaus, ACS Nano 2014, 8, 503-513.

[25] F. Pederzoli, G. Tosi, M. A. Vandelli, D. Belletti, F. Forni, B. Ruozi, Wiley Interdiscip. Rev. Nanomedicine Nanobiotechnology 2017, 9, 1-23.

[26] C. Weber, S. Morsbach, K. Landfester, Angew. Chemie - Int. Ed. 2019, 58, 12787- 
12794.

[27] H. Zhang, J. Peng, X. Li, S. Liu, Z. Hu, G. Xu, R. Wu, Colloids Surfaces $B$ Biointerfaces 2018, 167, 220-228.

[28] P. M. Kelly, C. Åberg, E. Polo, A. O’Connell, J. Cookman, J. Fallon, Ž. Krpetić, K. A. Dawson, Nat. Nanotechnol. 2015, 10, 472-479.

[29] N. Feiner-Gracia, M. Beck, S. Pujals, S. Tosi, T. Mandal, C. Buske, M. Linden, L. Albertazzi, Small 2017, 13, 1-11.

[30] A. M. Clemments, P. Botella, C. C. Landry, J. Am. Chem. Soc. 2017, 139, 39783981.

[31] A. C. G. Weiss, K. Krüger, Q. A. Besford, M. Schlenk, K. Kempe, S. Förster, F. Caruso, ACS Appl. Mater. Interfaces 2019, 11, 2459-2469.

[32] K. Welsher, H. Yang, Nat. Nanotechnol. 2014, 9, 198-203.

[33] H. Shen, L. J. Tauzin, R. Baiyasi, W. Wang, N. Moringo, B. Shuang, C. F. Landes, Chem. Rev. 2017, 117, 7331-7376.

[34] S. Hou, C. Johnson, K. Welsher, Molecules 2019, 24, DOI 10.3390/molecules24152826.

[35] S. Hou, X. Lang, K. Welsher, Opt. Lett. 2017, 42, 2390.

[36] S. Hou, J. Exell, K. Welsher, Nat. Commun. 2020, 1-33.

[37] M. P. Monopoli, D. Walczyk, A. Campbell, G. Elia, I. Lynch, F. B. Bombelli, K. A. Dawson, 2011, 2525-2534.

[38] G. F. and P. M. L. Vroman, A. Adams, Blood 1980, 55, 156-159.

[39] S. Tenzer, D. Docter, J. Kuharev, A. Musyanovych, V. Fetz, R. Hecht, F. Schlenk, D. Fischer, K. Kiouptsi, C. Reinhardt, K. Landfester, H. Schild, M. Maskos, S. K. Knauer, R. H. Stauber, Nat. Nanotechnol. 2013, 8, 772-781.

[40] V. P. Zhdanov, Curr. Opin. Colloid Interface Sci. 2019, 41, 95-103. 